\title{
Aminophosphines: a Double Role in the Synthesis of Colloidal Indi- um Phosphide Quantum Dots
}

\author{
Mickael D. Tessier, ${ }^{\dagger *}$ Kim De Nolf, ${ }^{\dagger+}$ Dorian Dupont, ${ }^{\dagger}$ Davy Sinnaeve, ${ }^{\Uparrow}$ Jonathan De Roo, ${ }^{\dagger}$ Zeger \\ Hens $^{\dagger *}$
}

†Physics and Chemistry of Nanostructures, Ghent University, Ghent, Belgium

†Center for Nano and Biophotonics, Ghent University, Ghent, Belgium

INMR and Structural Analysis Unit, Ghent University

\begin{abstract}
Aminophosphines have recently emerged as economical, easy-to-implement precursors for making InP nanocrystals, which stand out as alternative, Cd-free quantum dots for opto-electronic applications. Here, we present a complete investigation of the chemical reactions leading to InP formation starting from $\mathrm{InCl}_{3}$ and tris(dialkylamino)phosphines. Using nuclear magnetic resonance (NMR) spectroscopy and single crystal X-ray diffraction, we demonstrate that injection of the aminophosphine in the reaction mixture is followed by a rapid transamination with oleylamine, the solvent of the reaction. In addition, mass spectrometry and NMR indicate that the formation of InP concurs with that of tetra(oleylamino)phosphoniumchloride. The chemical yield of the InP formation agrees with this $4 \mathrm{P}(+\mathrm{III}) \rightarrow \mathrm{P}(-\mathrm{III})+3 \mathrm{P}(+\mathrm{V})$ disproportionation reaction occuring, since full conversion of the In precursor was only attained for a 4:1 P:In ratio. Hence the double role of the aminophosphine as both precursor and reducing agent. These new insights will guide further optimization of high quality InP quantum dots and might lead to the extension of synthetic protocols towards other pnictide nanocrystals.
\end{abstract}

\section{Introduction}

Colloidal nanocrystals have many applications ${ }^{1}$ ranging from opto-electronics $^{2-4}$ to energy ${ }^{5-8}$ and catalysis. ${ }^{9,10}$ In particular, quantum dots (QDs) fluorescing in the visible range are of significant interest for lighting and display applications. ${ }^{11-14} \mathrm{In}$ this respect, stringent restrictions on the use of cadmium in consumer products have initiated a shift from the wellcharacterized Cd-based QDs to Cd-free alternatives such as indium phosphide. The first synthesis of InP nanocrystals with a reasonable size-dispersion was reported in $1995 .{ }^{15}$ This synthesis employed tris(trimethylsilyl)phosphine (PTMS) which remains hitherto the most widely used phosphorus precursor for the synthesis of InP QDs. However, this compound is in many respects problematic as it is highly expensive and auto-ignites in contact with air. Recently, efficient protocols based on aminophosphines have been established to synthesize InP QDs of different sizes. ${ }^{16,17}$ As compared to PTMS, an aminophosphine precursor is inexpensive, safe-touse under ambient conditions and proved to lead to InP QDs of comparable quality as the best samples obtained with PTMS. ${ }^{18,19}$ In addition, this aminophosphine-based synthesis can lead to $\mathrm{InP} / \mathrm{ZnE}(\mathrm{E}=\mathrm{S}$ or $\mathrm{Se})$ core/shell QDs that emit from $500 \mathrm{~nm}$ to $670 \mathrm{~nm}$ with an emission linewidth between $44 \mathrm{~nm}$ and $65 \mathrm{~nm}$ and a photoluminescence quantum yield of 20 to $80 \% .^{17}$

Ever since the early days of Cd-based QD syntheses, insights in the reaction mechanism were used to improve the synthetic result in terms of size control and minimization of size dispersion. $^{20}$ By now, several QD synthesis methods have been shown to follow a two-step process where the injected precursors first react to form the solute or monomer whose increasing concentration subsequently gives rise to nucleation and growth of nanocrystals. ${ }^{21-23}$ In this respect, mechanistic studies have addressed both the chemical reactions involved in monomer formation and the relation between the monomer formation rate and the size of the resulting nanocrystals. ${ }^{24-27}$ Especially in the case of CdSe QDs synthesized using trioctylphosphine selenium (TOP-Se), precursor conversion was investigated in great detail and found to consist of two essential steps, i.e., a coordination of TOP-Se to the $\mathrm{Cd}$ center, followed by the cleavage of the $\mathrm{Se}=\mathrm{P}$ bond. Moreover, this precursor conversion was found to be limiting the overall rate of CdSe formation. ${ }^{27}$ This finding not only resulted in several size tuning strategies, ${ }^{24-26}$ it also indicates that a controlled synthesis of nanocrystals will only be possible if the precursor conversion is slow as compared to the nucleation and growth of the nanocrystals proper.

Importantly, such studies are shifting synthesis development from a semi-empirical trial-and-error approach to a more rational design of synthesis protocols that allows for optimal control of the QD size and size dispersion. A recent and most striking example involves the formation of lead sulfide QDs from thiourea precursors where insight in the relation between reaction rate and nanocrystal size was implemented to achieve size control at full reaction yield over a broad range of nanocrystal diameters. ${ }^{28}$ In the case of PTMS-based synthesis protocols for InP QDs, different mechanistic studies showed that precursor conversion is complete upon injection, ${ }^{29,30}$ resulting in ripening-driven nanocrystal growth. Although this was linked to the difficulty of synthesizing monodisperse InP QDs, the use of substituted silylphosphines with a lower reactivity did not overcome this problem. ${ }^{31}$ In the case of aminophosphine precursors on the other hand, the InP formation rate proved to be much slower. ${ }^{17}$ Whereas this lower rate was utilized to implement economical size-tuning-at-full-yield 
strategies, the chemistry of the conversion reaction is not yet understood. This, however, is crucial to further optimize the reaction and extend it to the synthesis of other metal phosphide and metal arsenide nanocrystals.

In this paper, we identify several key aspects that govern the formation of InP from aminophosphine precursors and indium chloride in the presence of primary amines. First, we show that the reaction involves the substitution of the original amino group by amines present in the reaction mixture, where only transamination with primary amines leads to InP formation. Furthermore, we find that the substituted aminophosphine has a double role in the reaction, both serving as the phosphorous precursor and the reducing agent by the formation of $\mathrm{P}(\mathrm{V})$ phosphonium salt. These findings are summarized in a chemical reaction mechanism that may help using this unique precursor chemistry for the formation of, e.g., GaP, InAs or GaAs nancrystals whose synthesis has proven even more chalenging than that of InP because of the bad size-dispersion ${ }^{15}$ or by the use of extremely hazardous precursor. ${ }^{32,33}$

\section{Experimental Section}

\section{Chemicals}

Indium(III) chloride (99.999\%), zinc(II) chloride ( $\geq 98 \%$ ), dodecylamine (99\%), tris(diethylamino)phosphine (97 \%), tris(dimethylamino)phosphine (97\%), selenium powder 100 mesh $(99.99 \%)$ and zinc stearate (technical grade, $65 \%$ ) were purchased from Sigma Aldrich. Trioctylphosphine (> $97 \%$ ) were purchased from Strem Chemicals. Oleylamine (80-90 \%) was purchased from Acros Organics (NB: Oleylamine is stored under inert atmosphere). Octadecene (technical $90 \%$ ) was purchased from Alfa Aesar.

\section{Synthesis of core/shell InP/ZnSe QDs}

$100 \mathrm{mg}$ (0.45 mmole) of indium(III) chloride, as indium raw materials and $300 \mathrm{mg}$ (2.2 mmole) of zinc(II) chloride, as zinc raw materials are mixed in $5.0 \mathrm{~mL}$ (15 mmole) of technical oleylamine which is a coordinating solvent. The reaction mixture is stirred and degassed at $120{ }^{\circ} \mathrm{C}$ for an hour and then heated to $180{ }^{\circ} \mathrm{C}$ under inert atmosphere. Upon reaching 180 ${ }^{\circ} \mathrm{C}$, a volume of $0.45 \mathrm{~mL}$ (1.6 mmole) of tris(diethylamino)phosphine are quickly injected in the above mixture. After the phosphorus precursor injection, the InP nanocrystals synthesis proceeded. The InP core QDs reaction occurs during 30 minutes. After 30 minutes, shell growth procedure starts: slow injection of $1 \mathrm{~mL}$ of stoichiometric trioctylphosphine-selenium (2.2 M). At 60 minutes: temperature is increased from $180{ }^{\circ} \mathrm{C}$ to $200{ }^{\circ} \mathrm{C}$. At 120 minutes: slow injection of $1 \mathrm{~g}$ of $\mathrm{Zn}$ (stearate $)_{2}$ in $4 \mathrm{~mL}$ of octadecene. Temperature is increased from $200{ }^{\circ} \mathrm{C}$ to $220{ }^{\circ} \mathrm{C}$. At 150 minutes: injection of $0.7 \mathrm{~mL}$ of stoichiometric trioctylphosphine-selenium (2.2 M). Temperature is increased from $220{ }^{\circ} \mathrm{C}$ to $240{ }^{\circ} \mathrm{C}$. At 180 minutes: slow injection of $1 \mathrm{~g}$ of $\mathrm{Zn}$ (stearate $)_{2}$ in $4 \mathrm{~mL}$ of octadecene. Temperature is increased from $240{ }^{\circ} \mathrm{C}$ to $280{ }^{\circ} \mathrm{C}$. At 210 minutes: slow injection of $0.7 \mathrm{~mL}$ of stoichiometric trioctylphosphineselenium $(2.2 \mathrm{M})$. Temperature is increased from $280{ }^{\circ} \mathrm{C}$ to $320{ }^{\circ} \mathrm{C}$. After 240 minutes: slow injection of $1 \mathrm{~g}$ of $\mathrm{Zn}$ (stearate $)_{2}$ in $4 \mathrm{~mL}$ of octadecene. 300 minutes: end of reaction. At the end of the reaction, the temperature is cooled down. InP/ZnSe nanocrystals are then precipitated in ethanol and suspended in chloroform.

\section{Mass-spectrometry}

InP core QDs are synthesized and precipitated with methanol. The supernatant is analyzed using a 6230 TOF-MS with ESIsource mass-spectrometer of Agilent Technologies in a range from 55 to 1160 g.mole ${ }^{-1}$.

\section{NMR spectroscopy}

Aliquots of 40-100 $\mu \mathrm{L}$ are taken from a synthesis and put into vials containing inert atmosphere (Argon). It is crucial to prevent contact between aliquots and air. Oxidation of the aminophosphine species into aminophosphine oxide species leads to the appearance of extra resonances in the NMR spectra that complicate the interpretation. A fixed volume of the aliquots is dissolved in $500 \mu \mathrm{L}$ toluene- $\mathrm{d}_{8}(99,50 \% \mathrm{D}$, purchased at Eurisotop) and transferred to an NMR tube (5 $\mathrm{mm}$ ). NMR measurements were recorded on a Bruker Avance III Spectrometer operating at a ${ }^{1} \mathrm{H}$ frequency of $500.13 \mathrm{MHz}$ and equipped with a BBI-Z probe or on a Bruker Avance II Spectrometer operating at a ${ }^{1} \mathrm{H}$ frequency of $500.13 \mathrm{MHz}$ and equipped with a TXI-Z probe (channels are $\left.{ }^{1} \mathrm{H},{ }^{13} \mathrm{C},{ }^{31} \mathrm{P}\right)$. The sample temperature was set to $298.15 \mathrm{~K}$. . Quantitative ${ }^{1} \mathrm{H}$ spectra were recorded with a $20 \mathrm{~s}$ delay between scans to allow full relaxation of all NMR signals. The quantification was done by using the Digital ERETIC method. ${ }^{31} \mathrm{P}$ spectra were recorded with a $20 \mathrm{~s}$ delay between scans to allow full relaxation of all NMR signals as well. Diffusion measurements (2D ${ }^{31} \mathrm{P}$ DOSY) were performed using a double stimulated echo sequence for convection compensation and with monopolar gradient pulses. ${ }^{34}$ Smoothed rectangle gradient pulse shapes were used throughout.

\section{Results and Discussion}
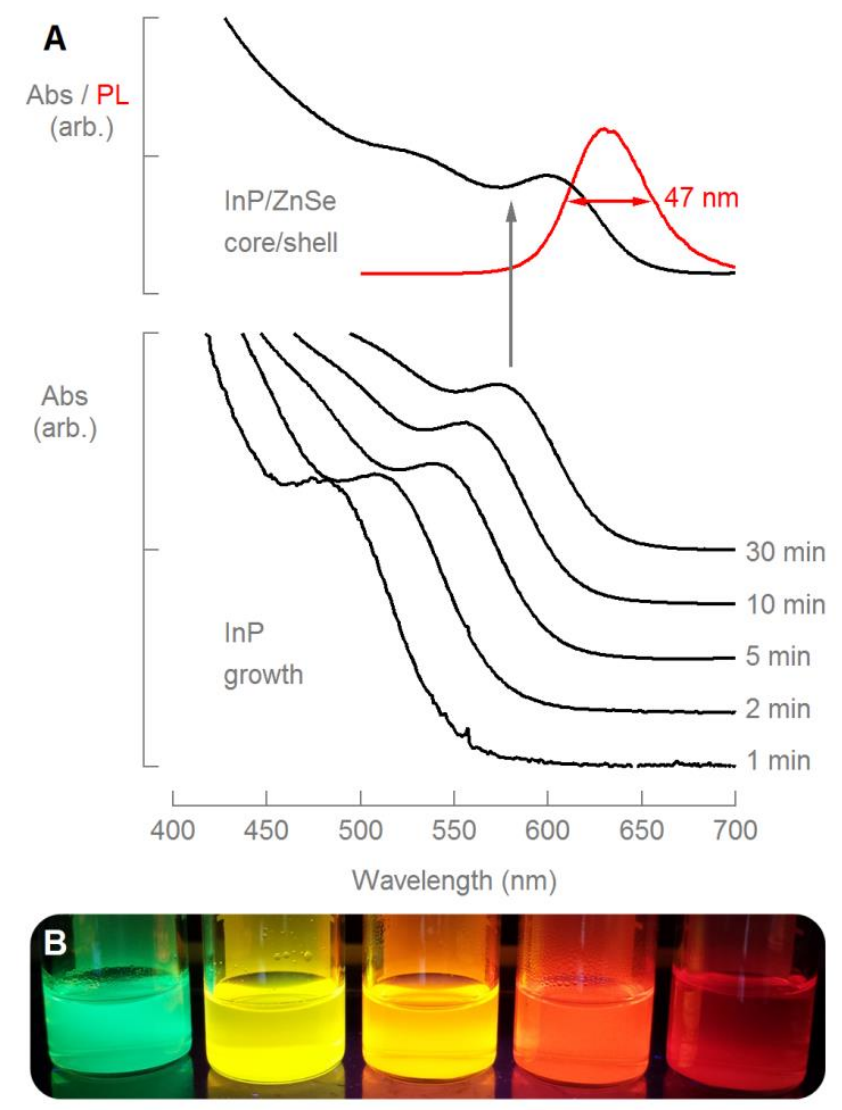

Figure 1: (A) (bottom) Development of the absorption spectrum of InP QDs during a typical aminophosphine-based synthesis of 
InP QDs and (top) absorption and photoluminescence spectra of resulting InP/ZnSe QDs. (B) Picture of a series of different $\mathrm{InP} / \mathrm{ZnE}$ QDs emitting from $500 \mathrm{~nm}$ to $670 \mathrm{~nm}$.

In a typical aminophosphine-based synthesis of InP QDs, indium halides and zinc halides are dissolved in a primary amine as solvent (e.g., oleylamine, $\left.\mathrm{OlNH}_{2}\right) .{ }^{16,17}$ The reaction mixture is subsequently degassed and heated to $150-220{ }^{\circ} \mathrm{C}$, followed by the injection of tris(dimethylamino)phosphine, $\mathrm{P}\left(\mathrm{NMe}_{2}\right)_{3}$, or tris(diethylamino)phosphine, $\mathrm{P}\left(\mathrm{NEt}_{2}\right)_{3}$. Figure $1 \mathrm{~A}$ depicts the UV-Vis absorption spectra of different aliquots taken at various reaction times for the case of an InP QD synthesis at $180{ }^{\circ} \mathrm{C}$ (see Experimental Section for details). Already after $1 \mathrm{~min}$ of reaction, the first excitonic feature is clearly visible and its red-shift with time points towards an increase of the QD diameter during the reaction. When the QDs reach their optimal size, shell precursors are added to the reaction mixture to obtain core/shell $\mathrm{InP} / \mathrm{ZnE}(\mathrm{E}=\mathrm{S}, \mathrm{Se}) \mathrm{QDs}$. In Figure 1A, the absorbance and photoluminescence spectra of InP/ZnSe QDs are shown. They have been obtained using stoichiometric trioctylphosphine selenium and zinc stearate mixed in octadecene as shell precursors (see Experimental Section for details). This sample exhibits a photoluminescence full width at half maximum of $47 \mathrm{~nm}$ and a photoluminescence quantum yield of $50 \%$ which are excellent values in comparison to the best core/shell InP/ZnE QDs obtained with PTMS precursor. ${ }^{18,19}$ Different size-tuning strategies, such as stopping the reaction before its end ${ }^{16}$ or using different indium halide or zinc halide precursors, ${ }^{17}$ allow obtaining InP/ZnE QDs over the entire visible range as shown in Figure 1B. A more extensive overview of absorbance and photoluminescence spectra of $\mathrm{InP} / \mathrm{ZnE}$ with emission varying from $500 \mathrm{~nm}$ to $670 \mathrm{~nm}$ and their associated synthesis protocols are presented in the Supplementary Information (see Supporting information S1).

A
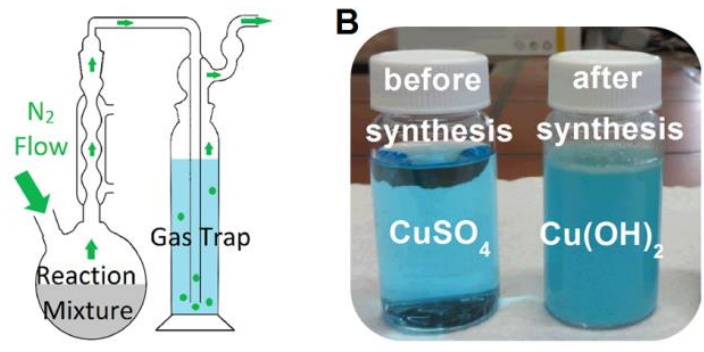

C

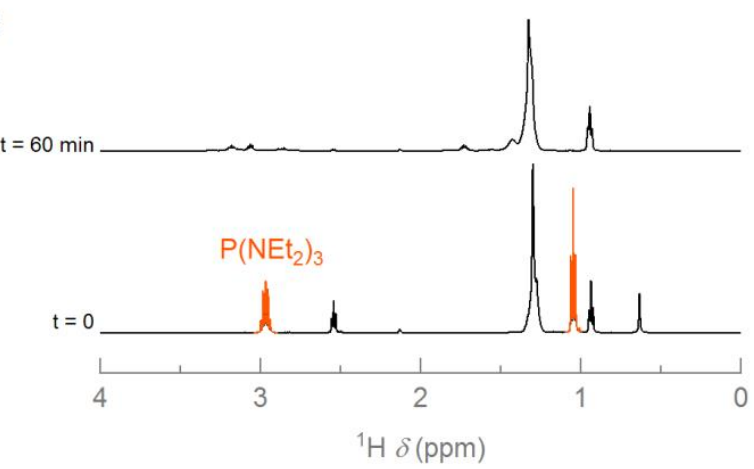

Figure 2: (A) Scheme of the gas trap mounting. (B) Picture of the $\mathrm{CuSO}_{4}$ gas trap aqueous solution before and after an InP synthesis. (C) ${ }^{1} \mathrm{H}$ NMR spectrum of $\mathrm{P}\left(\mathrm{NEt}_{2}\right)_{3}$ and dodecylamine, prior to and after reaction at $120^{\circ} \mathrm{C}$ for 60 minutes.
Although aminophosphines are excellent precursors for synthesizing InP QDs, they are not the most obvious. In PTMS, the phosphorus atom has an oxidation state of -III, rendering a reaction with an $\mathrm{In}(\mathrm{III})$ precursor such as indium chloride or indium acetate most likely. Aminophosphines on the other hand are $\mathrm{P}(+\mathrm{III})$ compounds, such that intermediate reduction steps are needed to form InP in a reaction with an In(III) precursor. Two-step methods in which at least the indium precursor is reduced before reacting with the phosphorus precursor have already been described in the literature. $\mathrm{InCl}_{3}$ for example can be reduced by $\mathrm{KBH}_{4}$ to form $\operatorname{In}(0)$ that then reacts with white phosphorus $\left(\mathrm{P}_{4}\right){ }^{35}$ Another example is the reduction of $\mathrm{InCl}_{3}$ by an organolithium reagent, followed by the reaction of the resulting $\operatorname{In}(0)$ with trioctylphosphine by a catalytic cleavage at high temperature. ${ }^{36}$ Opposite from aminophosphinebased syntheses, however, such two-steps strategies lead to rather polydisperse InP QDs. Alternatively, Song et al. hypothesized that InP formation starting from aminophosphine could involve the in-situ formation of phosphine $\left(\mathrm{PH}_{3}\right)$ due to the presence of labile hydrogen in the primary amine solvent. ${ }^{16}$ However, amines being poor Brønsted acids, such a mechanism seems unlikely.

To investigate possible intermediate reaction steps involved in the formation of InP out of aminophosphine and $\mathrm{InCl}_{3}$, we first scrubbed the exhaust of an InP QD synthesis using a chemical gas trap (Figure 2A). Most notably, we observed the formation of a copper hydroxide precipitate upon scrubbing with a saturated aqueous $\mathrm{CuSO}_{4}$ solution, a strong indication that the exhaust contains a basic gas (Figure 2B). Scrubbing with a methanolic solution of hydrogen chloride enabled us to trap the base as a salt. After rotary evaporation, a solid residue was obtained and after recrystallization in ethyl acetate, this salt was identified by single crystal X-ray diffraction as dimethylammonium chloride for a reaction where $\mathrm{P}\left(\mathrm{NMe}_{2}\right)_{3}$ was used as the phosphorus precursor (see Supporting Information S2). This points towards an exchange between the amines used as a solvent in the synthesis and the amino groups of the original precursor. This exchange is also observed using $\mathrm{P}\left(\mathrm{Net}_{2}\right)_{3}$ as the phosphorus precursor. Simply mixing $\mathrm{P}\left(\mathrm{NEt}_{2}\right)_{3}$ and dodecylamine in a vial under inert atmosphere confirms this transamination. Indeed, when the temperature is raised above 100 ${ }^{\circ} \mathrm{C}$, gas bubbles evolve, which we attribute to $\mathrm{Et}_{2} \mathrm{NH}$ rather than $\mathrm{P}\left(\mathrm{NEt}_{2}\right)_{3}$ as the latter has a boiling point of $245^{\circ} \mathrm{C}$. This is further confirmed by the two NMR spectra shown in Figure $2 \mathrm{C}$. The bottom one represents the mixture of $\mathrm{P}\left(\mathrm{NEt}_{2}\right)_{3}$ and dodecylamine prior to reaction. The resonances of $\mathrm{P}\left(\mathrm{NEt}_{2}\right)_{3}$ at 1.04 and $2.97 \mathrm{ppm}$, indicated in orange, can clearly be distinguished from the dodecylamine resonances in black. After one hour of reaction (Figure $2 \mathrm{C}$, top spectrum), the $\mathrm{P}\left(\mathrm{NEt}_{2}\right)_{3}$ resonances have vanished almost completely due to the diethylamino for dodecylamino exchange. Diethylamine is formed and evaporated from the mixture (boiling point $=55^{\circ} \mathrm{C}$ ). Since the spectra were recorded quantitatively, we can estimate the proportion of diethylamine that was evacuated from the mixture. In this particular case, after reaction at $120{ }^{\circ} \mathrm{C}$ for one hour, $97 \%$ of the original precursor is at least once transaminated.. Denoting an alkyl moiety in general as $\mathrm{R}$, this transamination can be written as a sequence of three successive reactions:

$\mathrm{P}\left(\mathrm{NEt}_{2}\right)_{3}+\mathrm{RNH}_{2} \rightleftharpoons \mathrm{P}\left(\mathrm{NEt}_{2}\right)_{2}(\mathrm{NHR})+\mathrm{Et}_{2} \mathrm{NH}$ 


$$
\begin{aligned}
& \mathrm{P}\left(\mathrm{NEt}_{2}\right)_{2}(\mathrm{NHR})+ \mathrm{RNH}_{2} \\
& \rightleftharpoons \mathrm{P}\left(\mathrm{NEt}_{2}\right)(\mathrm{NHR})_{2}+\mathrm{Et}_{2} \mathrm{NH} \\
& \mathrm{P}\left(\mathrm{NEt}_{2}\right)(\mathrm{NHR})_{2}+\mathrm{RNH}_{2} \rightleftharpoons \mathrm{P}(\mathrm{NHR})_{3}+\mathrm{Et}_{2} \mathrm{NH}
\end{aligned}
$$

Transamination reactions for $\mathrm{P}\left(\mathrm{NMe}_{2}\right)_{3}$ and $\mathrm{P}\left(\mathrm{NEt}_{2}\right)_{3}$ have been described before. ${ }^{37,38}$ Burgada for example argues that an exchange between the dimethylamino group of $\mathrm{P}\left(\mathrm{NMe}_{2}\right)_{3}$ and a heavier second amino group can occur if the hydrogen of this second amino group is sufficiently labile. ${ }^{37}$ As the boiling point of dimethylamine is $7.4^{\circ} \mathrm{C}$, this compound is eliminated from the reaction mixture after its formation, shifting the equilibrium of the reaction to the formation of a fully transaminated aminophosphine where the amino group has a higher boiling point than dimethylamine. Even if the author mentioned exchange between secondary amines only, it underlines our observation that a similar exchange involving a primary amine, such as dodecylamine or oleylamine, and a more volatile amino group, such as dimethylamino or diethylamino, occurs in the aminophosphine-based synthesis of InP.

\begin{tabular}{|c|c|c|}
\hline Class & Example & result \\
\hline & Butylamine & \\
& Octylamine & \\
& Dodecylamine & \\
& Oleylamine & \\
\hline $\begin{array}{c}\text { Secondary } \\
\text { amines }\end{array}$ & Dioctylamine & \\
\hline Tertiary amines & Trioctylamine & \\
\hline Other & Trioctylphosphine & \\
\hline
\end{tabular}

Table 1: Reaction results as a function of the solvent used.

The occurrence of transamination provides an important perspective on the role of the amino group in a particular aminophosphine precursor. First of all, it implies that adjusting this group to tune the precursor reactivity and thus the outcome of an InP synthesis risks to be an inefficient strategy if - as seems necessary - high boiling point amines are used as the solvent for the reaction. Indeed, in that case it will be the solvent that dictates what amino groups make up the true aminophosphine precursor. This explains for one thing why we do not observe significant differences in reactivity between, e.g., $\mathrm{P}\left(\mathrm{NMe}_{2}\right)_{3}$ and $\mathrm{P}\left(\mathrm{NEt}_{2}\right)_{3}$. On the other hand, the amino exchange highlights the role of the primary amine used as the solvent. By screening several amines, we find that InP is only formed when a primary amine is used. No reaction takes place in the case of secondary or tertiary amines (see Table 1 and also Supporting Information S). This clearly indicates that primary amines are not just the solvent or the ligand in an aminophosphine-based InP synthesis. Rather, they must play a central role in the whole precursor chemistry.

Focusing on syntheses using $\mathrm{P}\left(\mathrm{NEt}_{2}\right)_{3}$ and $\mathrm{InCl}_{3}$ in oleylamine, a key point to get further insight in the precursor conversion is the observation we already made before that the conversion yield of the indium precursor into InP, i.e. the chemical yield, depends on the aminophosphine vs. $\mathrm{InX}_{3}$ equivalence. ${ }^{17}$ Here, the chemical yield is calculated by comparing the absorbance of quantitative aliquots with the intrinsic absorption coeffi- cient of InP QDs at the same short wavelength. ${ }^{17}$ As shown in Figure 3, we find that the chemical yield increases proportionally to the $\mathrm{P}\left(\mathrm{NEt}_{2}\right)_{3}: \mathrm{InX}_{3}$ molar ratio to reach $100 \%$ for ratios of $\approx 4$ or more. At this point, we hypothesize that a phosphorus precursor excess is needed to obtain full chemical yield be-

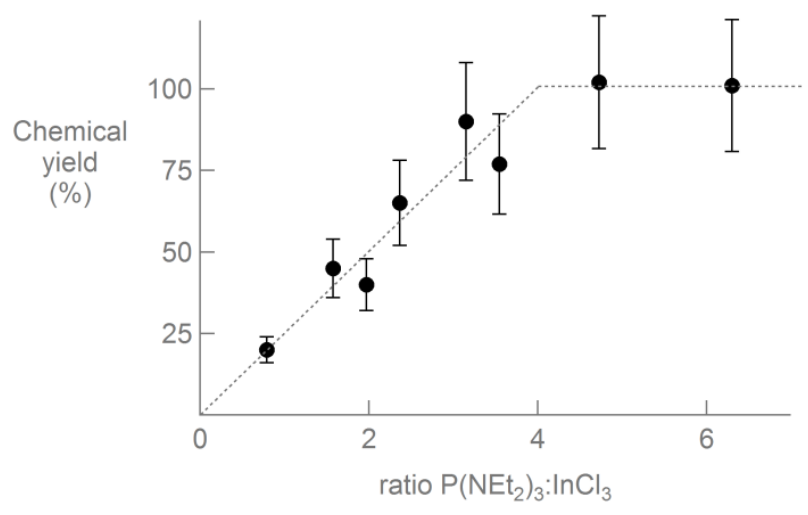

Figure 3: Conversion yield - amount of InP formed as fraction of the amount of $\mathrm{InCl}_{3}$ used - of a tris(diethylamino)phosphinebased InP QDs synthesis plotted as a function of the $\mathrm{P}\left(\mathrm{NEt}_{2}\right)_{3}: \mathrm{InCl}_{3}$ ratio used in the synthesis. The trend line is a straight line through the origin reaching full yield at a $\mathrm{P}\left(\mathrm{NEt}_{2}\right)_{3}: \mathrm{InCl}_{3}$ ratio of 4 , followed by a horizontal at $100 \%$ for higher $\mathrm{P}\left(\mathrm{NEt}_{2}\right)_{3} \mathrm{P}: \mathrm{InCl}_{3}$ ratios.

cause the aminophosphine plays more than one role in the synthesis. As discussed before, formation of InP from $\mathrm{P}\left(\mathrm{NEt}_{2}\right)_{3}$ comes with a reduction of $\mathrm{P}(\mathrm{III})$ to $\mathrm{P}(-\mathrm{III})$. One way to achieve this - and that accounts for the observed phosphorous excess needed to reach full conversion of the indium - is that one equivalent of phosphorous is reduced to $\mathrm{P}(-\mathrm{III})$ by the oxidation of 3 equivalents of phosphorous to $\mathrm{P}(+\mathrm{V})$. We have already demonstrated that injected $\mathrm{P}\left(\mathrm{NEt}_{2}\right)_{3}$ is modified during the synthesis by transamination. To continue this line of thought, we therefore propose an overall redox reaction in which 1 equivalent of $\mathrm{InP}$ is formed by the oxidation of 3 equivalents of the substituted aminophosphine to a phosphonium salt:

$\mathrm{InX}_{3}+4 \mathrm{P}(\mathrm{NHR})_{3} \rightarrow \operatorname{InP}+3 \mathrm{P}(\mathrm{NHR})_{4} \mathrm{X}$

In this chemical equation, $\mathrm{R}$ represents an alkyl moiety of a primary amine and $\mathrm{X}$ is either $\mathrm{Cl}, \mathrm{Br}$ or $\mathrm{I}$. 


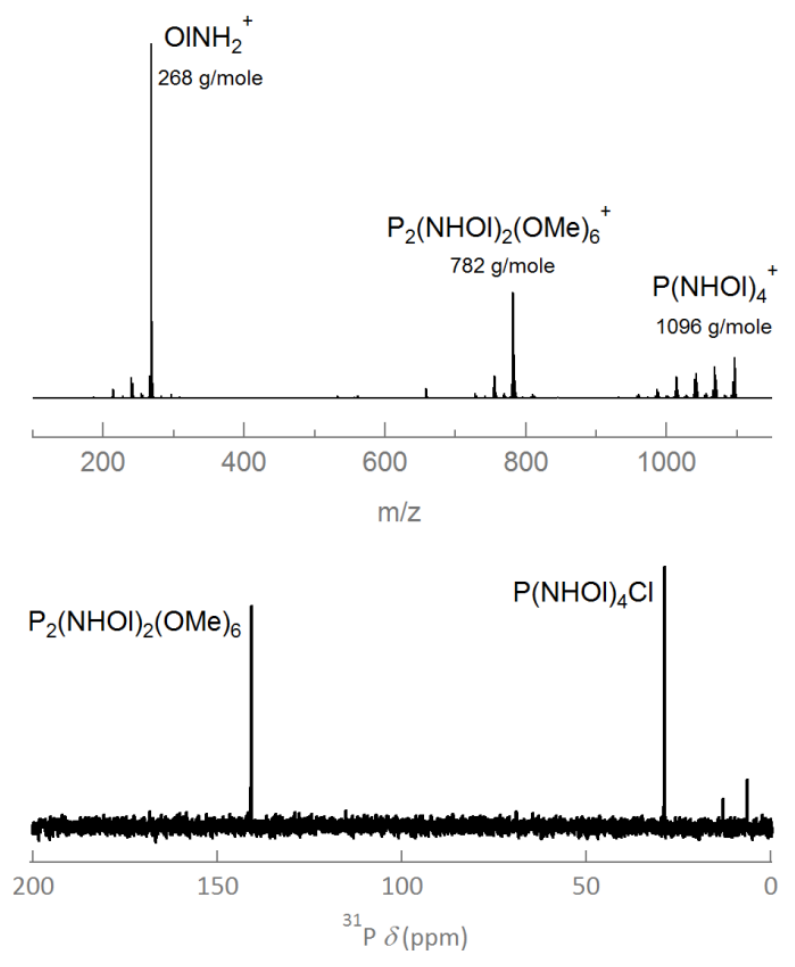

Figure 4: (A) Mass-spectrum of the supernatant obtained after purification by methanol addition of a full yield diethylaminophosphine-based InP QDs synthesis. (B) ${ }^{31} \mathrm{P}$ NMR spectrum of supernatant used for MS in toluene-d8.

First evidence that Eq. 4 indeed describes the overall reaction leading to InP comes from the mass spectrum of the reaction mixture after removal of the InP QDs by methanol addition. As shown in Figure 4, it contains three main compounds. A first has a molar mass of $268 \mathrm{~g} / \mathrm{mole}$, most likely corresponding to oleylamine, which is the solvent of the reaction and thus present in large amounts. The second compound has a molar mass of $782 \mathrm{~g} / \mathrm{mole}$, which corresponds to $\mathrm{P}_{2}(\mathrm{NHOl})_{2}(\mathrm{OMe})_{6}$. Probably, this has been produced during the purification in a reaction between aminophosphine compounds and methanol (see Experimental Section). The third compound has a measured molar mass of $1096 \mathrm{~g} / \mathrm{mole}$ which is close to the 1097 $\mathrm{g} /$ mole expected for $\mathrm{P}(\mathrm{NHOl})_{4}{ }^{+}$, i.e., the oleylamino phosphonium cation generated by the reaction according to Eq. 4 .

To investigate in more detail this InP QD synthesis, ${ }^{31} \mathrm{P}$ NMR spectroscopy has been used. It is a powerful tool for the reaction studied here as phosphorus compounds are essential elements of it. In Fig 5A, the ${ }^{31} \mathrm{P}$ NMR spectra of the precursor mixture and reaction aliquots are shown. Five resonances can be clearly distinguished. We identify the initial precursor at $118 \mathrm{ppm}$ since this compound can be measured as a pure substance. In addition, the resonance located at $29 \mathrm{ppm}$ is assigned to the phosphonium salt, since the ${ }^{31} \mathrm{P}$ NMR spectrum of the reaction supernantant (Fig 4B) already shows the location of this phosphonium salt resonance. The remainder of the resonances are most likely the transamination species. It was found in literature [ref] that these transamination species are located around $100 \mathrm{ppm}$ and exsist in a tautomeric prototropic equilibrium with iminophosphoranes (see scheme 1). These tautomeric forms are located around $5 \mathrm{ppm}$ with a ${ }^{1} \mathrm{~J}_{\mathrm{PH}}$ coupling of 500-600 Hz. Indeed, two resonances around $100 \mathrm{ppm}$ (111 and $98 \mathrm{ppm}$ ) and a third resonance at $13 \mathrm{ppm}$ are ob- served. The latter exhibits a ${ }^{1} \mathrm{~J}_{\mathrm{PH}}$ coupling of $550 \mathrm{~Hz}$ (see supporting information S). Unfortunately, it is very hard to assign these species to the different transamination products since they are chemically very equivalent. Furthermore, we link a diffusion coefficient as determined using diffusionordered spectroscopy (DOSY) to each of the main resonances in the precursor mixture or reaction aliquots (see also Supporting Information S3). Considering the transamination and phosphonium salt formation, this makes for a logical attribution of the resonance by linking smaller diffusion coefficients to more bulky species.<smiles>[R]N=[PH2](N[R])N[R]</smiles>

Scheme 1: Tautomeric prototropic equilibrium between aminophosphines and iminophosphoranes.

Having attributed the different ${ }^{31} \mathrm{P}$ resonances, the development of these different species can be monitored during a reaction by analyzing successive aliquots, where the concentration of each species is derived from the integrated intensity of its ${ }^{31} \mathrm{P}$ resonance. Taking a reaction carried out at $190{ }^{\circ} \mathrm{C}$ as an example, Figure $5 \mathrm{~A}$ and $5 \mathrm{~B}$ show that $15 \mathrm{sec}$ after injection, the transaminated compounds are already the prevailing species. Importantly, this transamination is considerably sped up by the presence of $\mathrm{InCl}_{3}$ and, especially, $\mathrm{ZnCl}_{2}$ (see Supporting Information S4). Moreover, the high rate of transamination as compared to the eventual InP formation points to a possible quasi-equilibrium between the different transaminated aminophosphines. Next, Figure 5B shows that during the reaction, the concentration of $\mathrm{P}(\mathrm{NHOl}){ }_{4} \mathrm{Cl}$ gradually increases at a rate concurring with the formation of InP with an approximately 3:1 equivalence. Clearly, this observation confirms the overall chemical reaction equation (4), where indeed one InP equivalent is formed together with three equivalents of $\mathrm{P}(\mathrm{NHOl})_{4} \mathrm{Cl}$ and it agrees with the $\mathrm{MS}$ analysis showing $\mathrm{P}(\mathrm{NHOl})_{4} \mathrm{Cl}$ to be the dominant reaction byproduct. The decrease in concentration of both transaminated aminophosphines follows the formation of InP. This could indicate that all transamination products are actual, reactive precursors. 

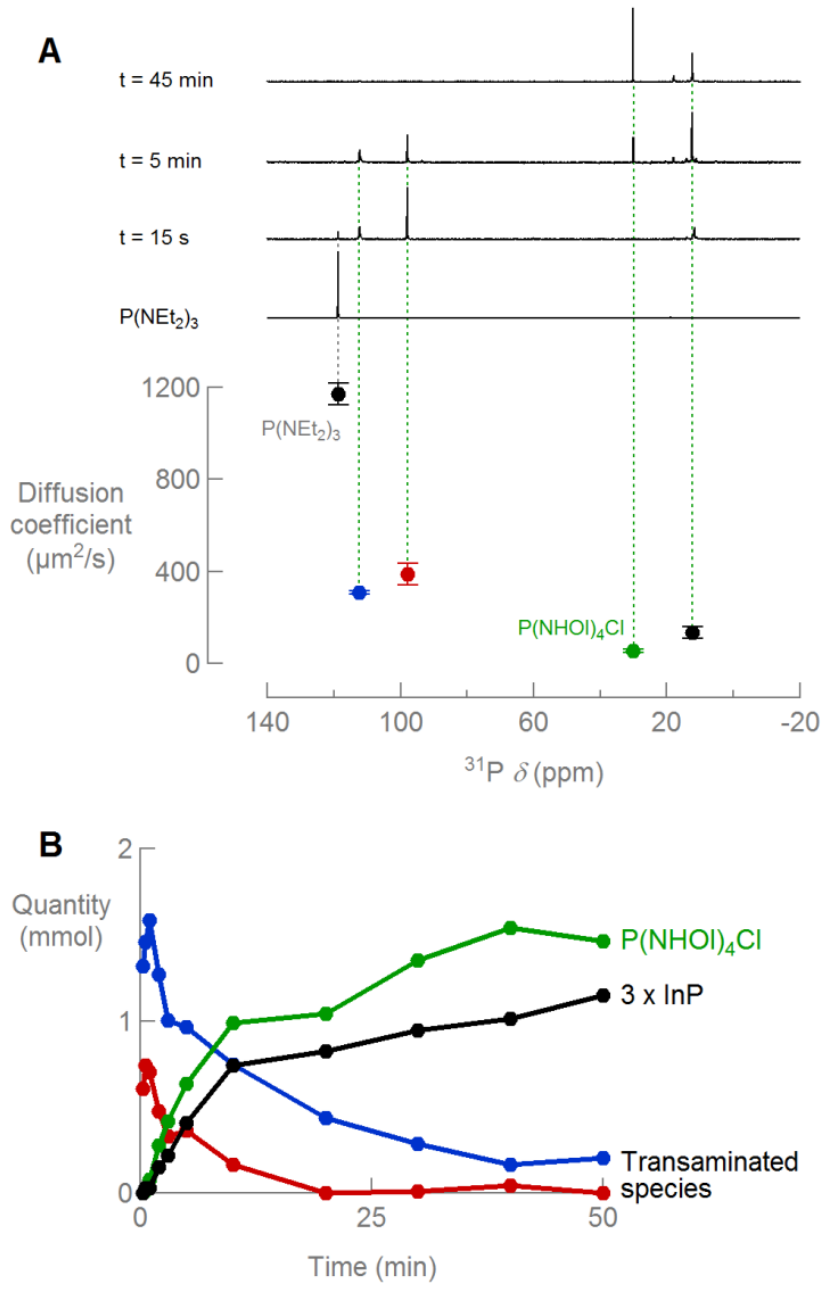

Figure 5: (A) (top) ${ }^{31} \mathrm{P}$ NMR spectra of $\mathrm{P}\left(\mathrm{NEt}_{2}\right)_{3}$ and different aliquots taken at the indicated time after initiating a diethylaminophosphine based InP synthesis run at $170{ }^{\circ} \mathrm{C}$, related to (bottom) the diffusion coefficient of the resonances as determined using ${ }^{31} \mathrm{P}$ DOSY. (B) (colored) Concentration development of the prevailing species during a diethylaminophosphine based InP synthesis run at $170{ }^{\circ} \mathrm{C}$ as estimated from ${ }^{31} \mathrm{P}$ NMR resonance intensities and (black) the concomitant amount of InP formed.

However, this seems flauwed given that $\mathrm{P}(\mathrm{NHOl})_{4} \mathrm{Cl}$ is the only reaction byproduct. As transamination seems restricted to compounds with a 3-coordinated trivalent phosphorous center ${ }^{38}$ the absence of partially substituted phosphonium salts indicates that indeed $\mathrm{P}(\mathrm{NHOl})_{3}$ is the only reactive phosphorous precursor. The concentration of the tautomeric form, on the other hand, stays largely constant. This is not surprising as the tautomeric form isn't reactive.

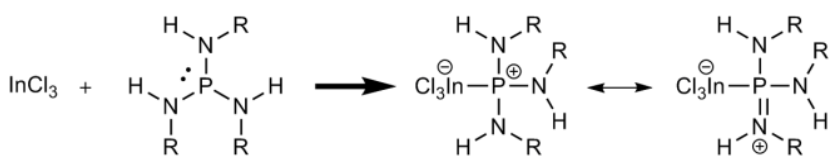

Scheme 2: In-P complex formation.

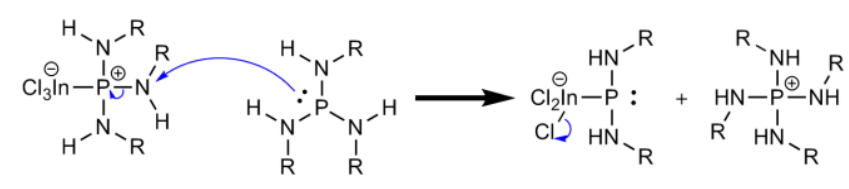

Scheme 3: Phosphorus nucleophilic substitution.

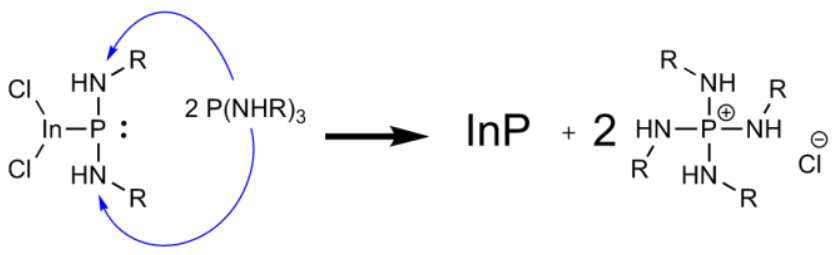

Scheme 4: InP unit formation.

Based on the overall InP formation reaction expressed by $\mathrm{Eq}$ (4), a chemical reaction mechanism can be proposed. In accordance with the double role of phosphorous, this mechanism assumes a nucleophile attack by the phosphorus center of one aminophosphine on an amino group of another aminophosphine. Considering that the fully transaminated $\mathrm{P}(\mathrm{NHR})_{3}$ is the actual reactant, scheme 2-4 represent the different steps this conversion involves. In a first step (scheme 2), an adduct of $\mathrm{InCl}_{3}$ and $\mathrm{P}(\mathrm{NHR})_{3}$ is formed. Importantly, such a complex exhibits different resonance structures (only two of which are shown), which lead to a delocalization of the positive charge over the phosphorous center and the surrounding nitrogen atoms. This renders the nitrogen atoms sensitive to a nucleophilic attack by a second $\mathrm{P}(\mathrm{NHR})_{3}$ as represented in scheme 3 . This results in the formation of an InP intermediate and a first equivalent of the $\mathrm{P}(\mathrm{NHR})_{4} \mathrm{Cl}$ phosphonium salt. Note that this reaction leads to a first change in the phosphorous oxidation numbers, with that in the InP intermediate being reduced from + III to + I and that in the phosphonium salt being oxidized from + III to $+\mathrm{V}$. Scheme 4 sketches subsequent reduction steps, where 2 more equivalents of $\mathrm{P}(\mathrm{NHR})_{3}$ further reduce the phosphorous in the InP intermediates to an oxidation state of I and -III, respectively and result in the formation of one equivalent of InP. The proposed pathway provides an explanation as to why InP is only formed when using primary amines as the solvent. Indeed, steric hindrance may hamper both the formation of the adduct depicted in scheme 2 and the subsequent nucleophilic attack on nitrogen. Moreover, an alkyl chain is an electron donating group that can further stabilize the positive charge in the adduct. This may render the nitrogen atoms less electrophilic, a necessary attribute for the subsequent reactions.

\section{Conclusion}

In summary, we have studied the precursor conversion in the aminophosphine-based synthesis of InP QDs. We have shown that upon injection, dimethyl- and diethylaminophosphine undergo rapid transamination with the amine used as the solvent. This leads to the release of dimethyl or diethylamine and an effective aminophosphine precursor that is the fully transaminated compound. Only in the case that primary amines are used, this leads to the eventual formation of $\mathrm{InP}$, where a quaternary aminophosphonium salt is formed as the major reaction byproduct. This finding enables the overall InP formation reaction to be written as a redox reaction where three equivalents of the transaminated aminophosphine reduce a fourth equivalent to form one equivalent of InP. Importantly, this double role of the aminophosphine explains why full conversion of the In precursor into InP is only attained with a 4-fold excess of the aminophosphine. This picture is confirmed by monitoring the reaction development using ${ }^{31} \mathrm{P}$ NMR spectroscopy. Based on these findings, we propose a chemical reaction mechanism that involves a nucleophilic 
attack of one aminophosphine on the nitrogen in a second aminophosphine that is activated by complexation with $\mathrm{InCl}_{3}$. We believe that the new chemistry introduced here for the formation of InP QDs may be extended to the formation of other III-V quantum dots, such as GaP, InAs or GaAs, using similar aminophosphine or aminoarsine precursors.

\section{ASSOCIATED CONTENT}

The supporting information includes InP/ZnS and InP/ZnSe QDs synthesis protocols, identification of dimethylamine gas by single crystal XRD, diffusion-ordered spectroscopy of the phosphorus prevailing species, comparison of the transamination rate for different protocols. This material is available free of charge via the Internet at http://pubs.acs.org.

\section{AUTHOR INFORMATION}

\section{Corresponding Author}

*mickael.tessier@ugent.be

*zeger.hens@ugent.be

\section{Present Addresses}

Krijgslaan 281, building S3 (Campus Sterre), 9000 Gent, Belgium.

\section{Author Contributions}

\$These authors contributed equally.

\section{Funding Sources}

This work was funded by Instituut voor de Aanmoediging van Innovatie door Wetenschap en Technologie in Vlaanderen (IWTSBO Lumicor). Z.H. acknowledges support by the European Comission via the Marie-Sklodowska Curie action Phonsi (H2020-MSCA-ITN-642656), the Belgian Science Policy office (IAP 7.35, photonics@be) and Ghent University (GOA 01G01513) for funding. J. D. R., K. D. N. and D. S. thank the FWO for their fellowships.

\section{Notes}

The authors declare no competing financial interests.

\section{ACKNOWLEDGMENT}

The authors are grateful to: prof. J. Martins for use of the NMR spectrometers, prof. K. Van Hecke for the single-crystal diffraction, and prof. F. Lynen for assistance with the mass spectrometry.

\section{ABBREVIATIONS}

QDs, Quantum Dots; PTMS, tris(trimethylsilyl)phosphine; $\mathrm{P}\left(\mathrm{NMe}_{2}\right)_{3}$, tris(dimethylamino)phosphine; $\quad \mathrm{P}\left(\mathrm{NEt}_{2}\right)_{3}$, tris(dimethylamino)phosphine; $\mathrm{R}$, alkyl; Ol, oleyl $\left(\mathrm{C}_{18} \mathrm{H}_{35}\right)$.

\section{REFERENCES}

(1) Kovalenko, M. V; Manna, L.; Cabot, A.; Hens, Z.; Talapin, D. V; Kagan, C. R.; Klimov, X. V. I.; Rogach, A. L.; Reiss, P.; Milliron, D. J.; Guyot-sionnnest, P.; Konstantatos, G.; Parak, W. J.; Hyeon, T.; Korgel, B. A.; Murray, C. B.; Heiss, W. ACS Nano 2015, 9, 1012.

(2) Klimov, V. I. Science (80-. ). 2000, 290, 314

(3) Semonin, O. E.; Luther, J. M.; Choi, S.; Chen, H.; Gao, J.; Nozik, A. J.; Beard, M. C. Science (80-. ). 2011, 1530, 1530.

(4) Wang, X.; Koleilat, G. I.; Tang, J.; Liu, H.; Kramer, I. J.; Debnath, R.; Brzozowski, L.; Barkhouse, D. A. R.; Levina, L.; Hoogland, S.; Sargent, E. H. Nat. Photonics 2011, 5, 480.
(5) Cadavid, D.; Ibáñez, M.; Gorsse, S.; López, A. M.; Cirera, A.; Morante, J. R.; Cabot, A. J. Nanoparticle Res. 2012, $14,1$.

(6) Ibáñez, M.; Korkosz, R. J.; Luo, Z.; Riba, P.; Cadavid, D.; Ortega, S.; Cabot, A.; Kanatzidis, M. G. J. Am. Chem. Soc. 2015, 137, 4046.

(7) Oszajca, M. F.; Bodnarchuk, M. I.; Kovalenko, M. V. Chem. Mater. 2014, 26, 5422.

Oh, M. H.; Yu, T.; Yu, S.-H.; Lim, B.; Ko, K.-T.; Willinger, M.G.; Seo, D.-H.; Kim, B. H.; Cho, M. G.; Park, J.-H.; Kang, K.; Sung, Y.-E.; Pinna, N.; Hyeon, T. Science (80-. ). 2013, 340, 964.

(9) De Roo, J.; Van Driessche, I.; Martins, J. C.; Hens, Z. Nat. Mater. 2016.

(10) Shylesh, S.; Schünemann, V.; Thiel, W. R. Angew. Chemie - Int. Ed. 2010, 49, 3428.

(11) Bourzac, K. Nature 2013, 493, 283.

(12) Chen, O.; Zhao, J.; Chauhan, V. P.; Cui, J.; Wong, C.; Harris, D. K.; Wei, H.; Han, H.-S.; Fukumura, D.; Jain, R. K.; Bawendi, M. G. Nat. Mater. 2013, 12, 445.

(13) Rizzo, A.; Li, Y.; Kudera, S.; Della Sala, F.; Zanella, M.; Parak, W. J.; Cingolani, R.; Manna, L.; Gigli, G. Appl. Phys. Lett. 2007, 90, 2.

(14) Bae, W. K.; Park, Y.-S.; Lim, J.; Lee, D.; Padilha, L. a; McDaniel, H.; Robel, I.; Lee, C.; Pietryga, J. M.; Klimov, V. I. Nat. Commun. 2013, 4, 2661.

(15) Micic, O. I.; Sprague, J. R.; Curtis, C. J.; Jones, K. M.; Machol, J. L.; Nozik, A. J.; Giessen, H.; Fluegel, B.; Mohs, G.; Peyghambarian, N. J. Phys. Chem. 1995, 99, 7754.

(16) Song, W.-S.; Lee, H.-S.; Lee, J. C.; Jang, D. S.; Choi, Y.; Choi, M.; Yang, H. J. Nanoparticle Res. 2013, 15, 1750.

(17) Tessier, M. D.; Dupont, D.; De Nolf, K.; De Roo, J.; Hens, Z Chem. Mater. 2015, 27, 4893.

(18) Xie, R.; Battaglia, D.; Peng, X. J. Am. Chem. Soc. 2007, 129, 15432.

(19) Li, L.; Reiss, P. J. Am. Chem. Soc. 2008, 130, 11588

(20) Murray, C. B.; Norris, D. J.; Bawendi, M. G. J. Am. Chem. Soc. 1993, 115, 8706.

(21) Deng, Z.; Cao, L.; Tang, F.; Zou, B. J. Phys. Chem. B 2005, 109, 16671.

(22) Steckel, J. S.; Yen, B. K. H.; Oertel, D. C.; Bawendi, M. G. J. Am. Chem. Soc. 2006, 128, 13032.

(23) Liu, H.; Owen, J. S.; Alivisatos, a. P. J. Am. Chem. Soc. 2007, 129, 305.

(24) Abe, S.; Capek, R. K.; De Geyter, B.; Hens, Z. ACS Nano 2012, 6,42 .

(25) Abe, S.; Capek, R. K.; De Geyter, B.; Hens, Z. ACS Nano 2013, 7, 943.

(26) De Nolf, K.; Capek, R. K.; Abe, S.; Sluydts, M.; Jang, Y.; Martins, J. C.; Cottenier, S.; Lifshitz, E.; Hens, Z. J. Am. Chem. Soc. 2015, 137, 2495-2505.

(27) Owen, J. S.; Chan, E. M.; Liu, H.; Alivisatos, A. P. J. Am Chem. Soc. 2010, 132, 18206.

(28) Hendricks, M. P.; Campos, M. P.; Cleveland, G. T.; Jen-La Plante, I.; Owen, J. S. Science (80-. ). 2015, 348, 1226. Allen, P. M.; Walker, B. J.; Bawendi, M. G. 2010, 760 Gary, D. C.; Cossairt, B. M. Chem. Mater. 2013, 25, 2463. Gary, D. C.; Glassy, B. A.; Cossairt, B. M. Chem. Mater. 2014, 26, 1734.

(32) Guzelian, A. A.; Banin, U.; Kadavanich, A. V; Peng, X.; Alivisatos, A. P. Appl. Phys. Lett. 1996, 69, 1432.

(33) Cao, Y. W.; Banin, U. J. Am. Chem. Soc. 2000, 122, 9692.

(34) Connell, M. A.; Bowyer, P. J.; Adam Bone, P.; Davis, A. L.; Swanson, A. G.; Nilsson, M.; Morris, G. A. J. Magn. Reson. 2009, 198, 121.

(35) Yan, P.; Xie, Y.; Wang, W.; Liu, F.; Qian, Y. J. Mater. Chem. 1999, 9,1831

(36) Lauth, J.; Strupeit, T.; Kornowski, A.; Weller, H. Chem. Mater. 2013, 25, 1377.

(37) Burgada, R. Bull. Soc. Chim. Fr. 1963, 10, 2335.

(38) Gopalakrishnan, J. Appl. Organomet. Chem. 2009, 23, 291. 


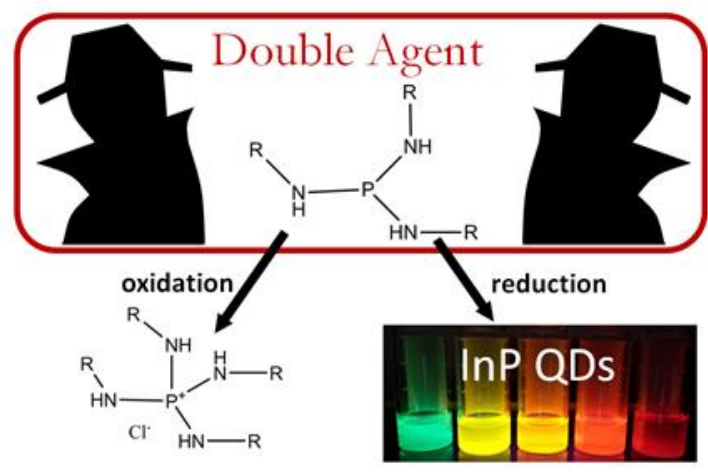

